\title{
Environmental impact of saltwater in a stratified flow system and its remedial measure
}

\author{
H. N. Hashmi ${ }^{1}$, N. E. Malik ${ }^{1}$ \& G. A. Hamill ${ }^{2}$ \\ ${ }^{l}$ Civil Engineering Department, University of Engineering \& Technology, \\ Taxila, Pakistan \\ ${ }^{2}$ Queens University of Belfast, Northern Ireland, UK
}

\begin{abstract}
Due to the improper mixing of saline water and freshwater within the estuary mouth stratification occurs. The weir obstacle in front of the estuary mouth forms an entrapped saline wedge. When dissolved, oxygen in the saline wedge goes behind a permissible limit and it endangers marine life. The anaerobic decomposition of organic matter at the bottom results in noxious environmental conditions. The same types of problems are being faced at River Lagan bay in Northern Ireland. This paper discusses the application of propeller washes on a stratified flow system to develop a technique to eliminate saltwater. Conductivity, velocity and temperature at different points along the depth in a stratified flow system were observed in detail during this research. Using the flume arrangement an artificial stratified condition so close to natural conditions was produced and tested in the laboratory under different sets of experiments. From the experimental results it was found that the propeller rotating at a higher speed was more effective at mixing stratified layers than that rotating at a lower speed. Experimental results also showed that an increase in propeller-tip clearance from the saltwater layer reduced the rate of erosion of saltwater.
\end{abstract}

Keywords: arrested saline wedge, entrapped saline wedge, multi-wire conductivity probe, flow stratification, stratified flow flume.

\section{Introduction}

Mixing of freshwater and saltwater is a complex process because of two chemically and physically different water bodies that come into contact with each other. Within estuaries, three different mixing regimes have been identified: 
(i) stratified, (ii) partially mixed, and (iii) mixed or homogenous. Stratified estuaries occur when the velocity of the discharging freshwater river is below a critical velocity at which mixing between the freshwater and saltwater normally occurs and the lighter freshwater overrides the denser saltwater layer; frictional forces along the density interface then erode the underlying saltwater into a wedge shape. The term arrested saline wedge is used to describe this situation. When an obstacle such as weir is placed across an estuary mouth, it results in a different type of saltwater wedge whose tip is isolated from its main ocean source. Such a wedge is known as an entrapped saline wedge.

Dissolved oxygen within the lower layer is more important for the survival of marine life. Entrapped saline wedge can get oxygen only from the overlying freshwater. However density stratification reduces mixing, and penetration of dissolved oxygen. Anaerobic condition at bottom causes decomposition of the organic mud and this may lead to the emission of ill-smelling hydrogen sulphide gas due to the presence of sulphates within the mud. The mixing which can avoid this situation is possible naturally when the overflowing freshwater has a velocity large enough to break the interface between the two layers. Artificial devices such as bubble diffusers have been employed to break up the stratification but found inefficient to break the interface. Modern weir and barrage designs such as Lagan Weir (N. Ireland) also incorporate the low-level withdrawal pipes that permit for the extraction of saltwater at low tide.

Keulegan [1] developed models to predict the shape of the arrested saline wedge, It was established by Arita and Jirka [2] that the stratified flows consist of two layers only with the formation of the third, or interfacial layer ignored due to its size in relation to the other layers. They further introduced the concept of Zero Velocity Line. The presence of the interfacial layer was recognised by Grubert [3], Sorgard [4], Curran [5], and Walker [6].

The literature was thoroughly reviewed but no work was found related to the use of propeller washes to break the interface in a density stratified flow system. The current investigation aims to apply different range of propeller washes on an entrapped saline wedge to break the interface and allow the mixing of saltwater with overflowing freshwater. This research may help to achieve a sustainable river system.

\section{Experimental apparatus and procedure}

Following experimental facility was arranged to investigate the problem.

\subsection{Stratified flow flume}

An experimental flume, $19.5 \mathrm{~m}$ long with cross-sectional dimensions $750 \mathrm{~mm} \mathrm{x}$ $750 \mathrm{~mm}$, was used as shown in Fig. 1. Experiments were performed on a saltwater impoundment of depth $250 \mathrm{~mm}$ with the overflowing freshwater of depth $400 \mathrm{~mm}$. Conductivity, temperature and velocity meters and pump controls were mounted in two steel cabinets beside the flume. One cabinet housed the pump controls for regulating pump speeds and the overflow alarms used to 
prevent flooding. The second cabinet held the conductivity meters and magmeter to display flow through the system. Freshwater was circulated around the flume with the use of a large capacity electric pump.

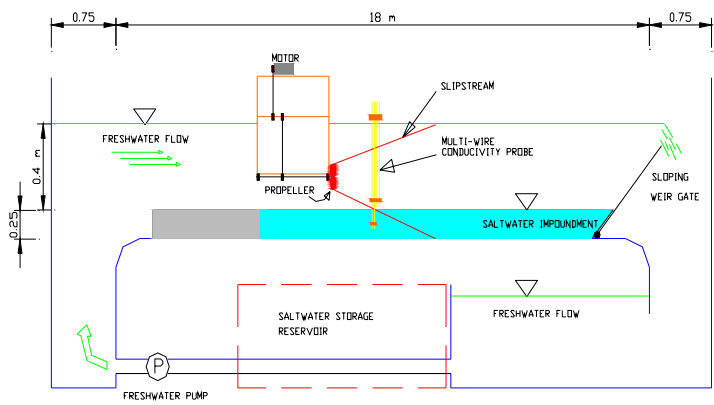

Figure 1: $\quad$ Stratified flow flume.

\subsection{Propeller drive system}

In order to generate a range of screw washes, a rig was designed and manufactured. An angle-iron frame was made along with a D.C. electric motor with variable speed control, rated at $0.55 \mathrm{~kW}$, with a maximum speed of $800 \mathrm{rpm}$ was fitted at the top. Three shafts were fitted on the rig, i.e. a motor shaft, an intermediate shaft and a propeller shaft. These shafts were connected through a gearing system consisting of two belts and four pulleys. This arrangement allowed the propeller to run at the required revolutions i.e. varying from $0-1600$ rpm.

\subsection{Multi-wire conductivity probe and its calibration}

A multi-wire conductivity probe consisting of four wires was manufactured. Each of the probes was shielded with an insulating plastic and connected through a lead to a separate conductivity meter located in the steel cabinet besides the flume. Probes measure conductivity in mili Siemens $(\mathrm{mS})$. The multi-wire conductivity probes were calibrated as the surrounding walls affect the value of conductivity reported. Thus the full length of the saltwater impoundment was used for calibration.

\section{Testing procedure}

The flume was filled with freshwater to a depth of $250 \mathrm{~mm}$. The measured amount of salt was added to make a solution of the required density. The solution was thoroughly mixed by stirring with a big brush. The samples of the solution at 
different location within the impoundment were taken to ensure uniform mixing of the solution. After achieving the required density within the saltwater impoundment, the flume was very slowly filled with freshwater to avoid any mixing with the underlying saltwater. The freshwater pumping system is capable of returning flow back into the freshwater reservoir by using return valve. Then pump was turned on to get the required speed. The return valve was then slowly adjusted to achieve the required freshwater velocity in the flume.

Conductivity probes were set at depths $50 \mathrm{~mm}, 200 \mathrm{~mm}, 300 \mathrm{~mm}$ and 450 $\mathrm{mm}$ from the base of the flume. These points were chosen on a vertical section at a horizontal distance equal to $2.5 \mathrm{Dp}$ from the face of the propeller. Initial conductivity reading for each probe was noted down. The propeller was allowed to run at the required speed and the conductivity values for each of the probes were recorded at increasing time intervals. Readings were taken at every 20 second for first five minutes. Then this interval was increased to 1 minute and the readings were noted for the period from 5 to 10 minutes. The interval was again increased to 5 minutes to measure conductivity values up to 30 minutes. An interval of 15 minutes was maintained for taking readings between 30 to 60 minutes. The next intervals were then increased to double of the previous ones, i.e. $1 / 2$ hour, 1 hour, 2 hours, 4 hours and so on until the test was completed. The test at low speed took nearly 40 hours to scour the saltwater layer fully.

\section{Testing programme}

After carrying out a number of preliminary tests, the actual testing programme was started. The current study aimed to investigate the influence of propeller wash on the erosion of saltwater in a stratified flow system. In order to study the propeller wash effects distinctly, all the parameters related to the stratified flow system were kept constant. The parameters related to the stratified flow system were as follows: -

$\begin{array}{ll}\text { Freshwater Velocity } & 20 \mathrm{~mm} / \mathrm{s} \\ \text { Freshwater Flow } & 6 \mathrm{lit} / \mathrm{s} \\ \text { Freshwater Depth } & 400 \mathrm{~mm} \\ \text { Saltwater Density } & 1032 \mathrm{~kg} / \mathrm{m}^{3} \text { (Irish seawater) } \\ \text { Saltwater Depth } & 250 \mathrm{~mm}\end{array}$

The propeller was rotated at three different speeds, i.e. $100 \mathrm{rpm}, 200 \mathrm{rpm}$ and $300 \mathrm{rpm}$ by keeping the propeller tip to saltwater surface clearance at $85 \mathrm{~mm}$. For each speed the conductivity readings for all the probes were recorded from the conductivity meters. These readings were then converted to density values prior to analysis. To investigate the problems clearly tests were also conducted for different propeller-tip clearances from the saltwater surface. For these tests, the propeller was rotated at a constant speed of $200 \mathrm{rpm}$. Again the conductivity values were noted from the conductivity meters and converted to corresponding density readings before any data manipulation. 


\section{Results and analysis}

A number of preliminary tests were conducted before the start of actual testing programme. These initial tests were employed to refine the experimental techniques to ensure all the experimental equipment would be operated precisely. The validity of the data acquisition system was also checked. Several colour visualisation tests were carried out which shows the initial lifting of red dyed saltwater under the action of the propeller wash. Once the saltwater impoundment eroded to a depth $50 \mathrm{~mm}$ from the base of the flume, the test was stopped. The rate of erosion of the saltwater impoundment was mainly dependent upon the propeller speed. The shortest experimental test lasted approximately 2 hours for a propeller speed of $300 \mathrm{rpm}$ and the longest test was completed in 40 hours for a propeller speed of $100 \mathrm{rpm}$.

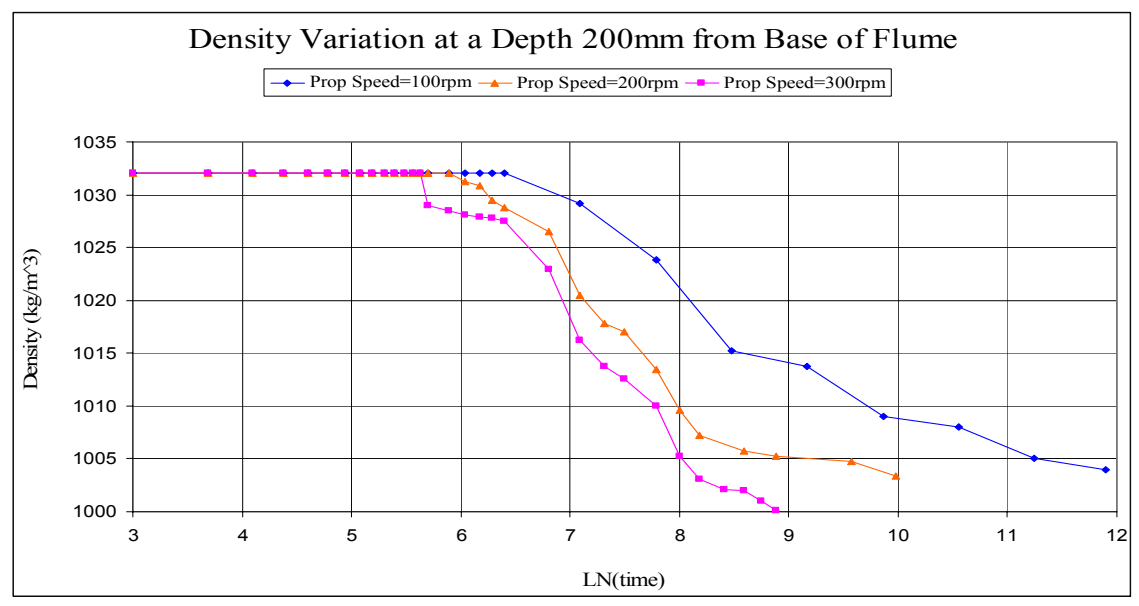

Figure 2: Density variation at a depth $200 \mathrm{~mm}$ from base of flume.

\subsection{Influence of propeller speed on saltwater erosion}

In order to investigate the effect of propeller speed on the erosion of saltwater layer, the propeller was rotated at three different speeds, i.e. $100 \mathrm{rpm}, 200 \mathrm{rpm}$ and $300 \mathrm{rpm}$. Figure 2 shows density variation with time at a depth $200 \mathrm{~mm}$ from the base, i.e. $50 \mathrm{~mm}$ below the interface of freshwater and saltwater. Logarithm values of time intervals were plotted on horizontal axis while the density readings were represented by the vertical axis. This figure shows that for propeller speed of $100 \mathrm{rpm}$, the scouring of saltwater was started after a period of 20 minutes and the density was reduced to $1004 \mathrm{~kg} / \mathrm{m}^{3}$ in 40 hours. For propeller speed of $200 \mathrm{rpm}$, the saltwater erosion started after 7 minutes and the density reading was observed to be $1003 \mathrm{~kg} / \mathrm{m}^{3}$ after 6 hours. The density started to decrease only after 5 minutes for the propeller speed of $300 \mathrm{rpm}$ and density reduction rate for this speed was very rapid and the saltwater diluted to 1000 $\mathrm{kg} / \mathrm{m}^{3}$ in approximately 2 hours. From all these curves, it can be concluded that 
an increase in propeller speed corresponds to a higher rate of density reduction. It happens because at a higher propeller speed, the wash produces larger turbulent currents lifting additional saltwater to mix with the overflowing freshwater. As a result, the saltwater density reduces at a faster rate.

Figure 3 reveals a plot of density readings vs. logarithm of time values at a depth $300 \mathrm{~mm}$ from the base of the flume. The initial value of density was 1000 $\mathrm{kg} / \mathrm{m}^{3}$ as this depth occurred in the freshwater. When the test was started, the saltwater lifted and mixed with the overflowing freshwater. For the propeller speed of $100 \mathrm{rpm}$, the maximum density was observed after 10 hours 40 minutes and it was $1004 \mathrm{~kg} / \mathrm{m}^{3}$. Then the density started reducing and reached a value $1000.20 \mathrm{~kg} / \mathrm{m}^{3}$ in 40 hours. Reduction in density at this point happened because the rate of dilution due to the incoming freshwater became more than the rate of concentration by the saltwater. The same behaviour was observed for the propeller speeds 200 and $300 \mathrm{rpm}$. For $200 \mathrm{rpm}$ speed, the maximum value of density reached to $1010.7 \mathrm{~kg} / \mathrm{m}^{3}$ after 2 hours while for $300 \mathrm{rpm}$ speed, it attained a value of $1017 \mathrm{~kg} / \mathrm{m}^{3}$ after 50 minutes. It can be seen in the graphs that rate of density increase for a higher speed is more than that for a lower speed of propeller.

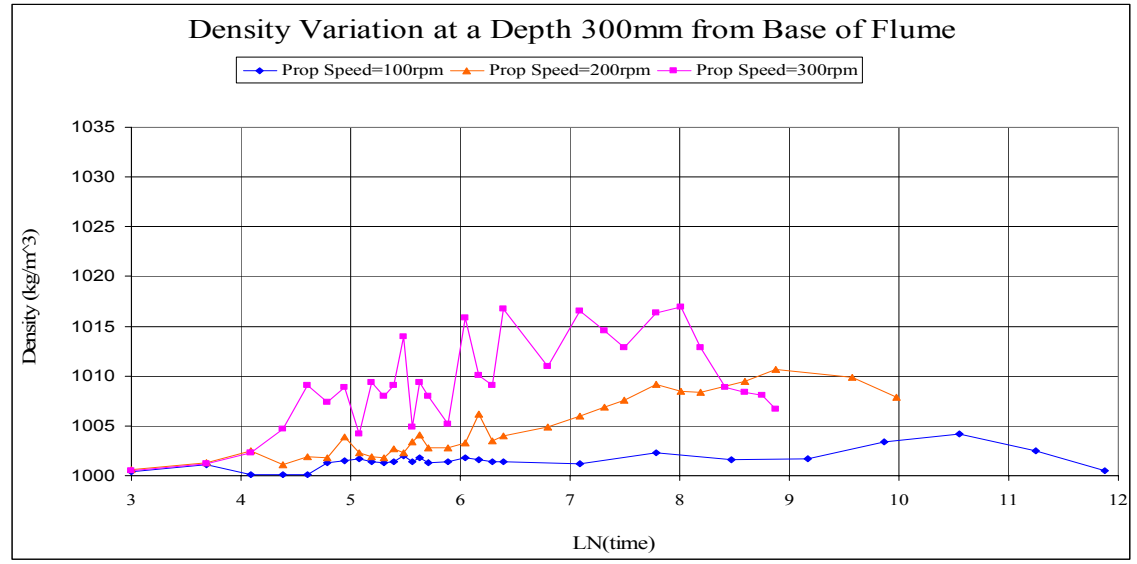

Figure 3: $\quad$ Density variation at a depth of $300 \mathrm{~mm}$ from base of flume.

The variations in the density of freshwater flow for the depth $450 \mathrm{~mm}$ are shown in Fig. 4. For propeller speeds 100, 200 and $300 \mathrm{rpm}$, the maximum density values attained were $1002.3,1005$ and $1012.5 \mathrm{~kg} / \mathrm{m}^{3}$ after the time intervals 4 hours, 17.5 minutes and 10 minutes respectively.

By comparing density values of Fig. 3 and Fig 4 it can be observed that the density readings are less at depth $450 \mathrm{~mm}$ than those at $300 \mathrm{~mm}$. It happened because the intensity of turbulent eddies, lifting the saltwater in upward direction was less at depth $450 \mathrm{~mm}$ than that at depth $300 \mathrm{~mm}$. Comparing both the figures, it can be noticed that the density variations followed a similar trend in both the cases. 


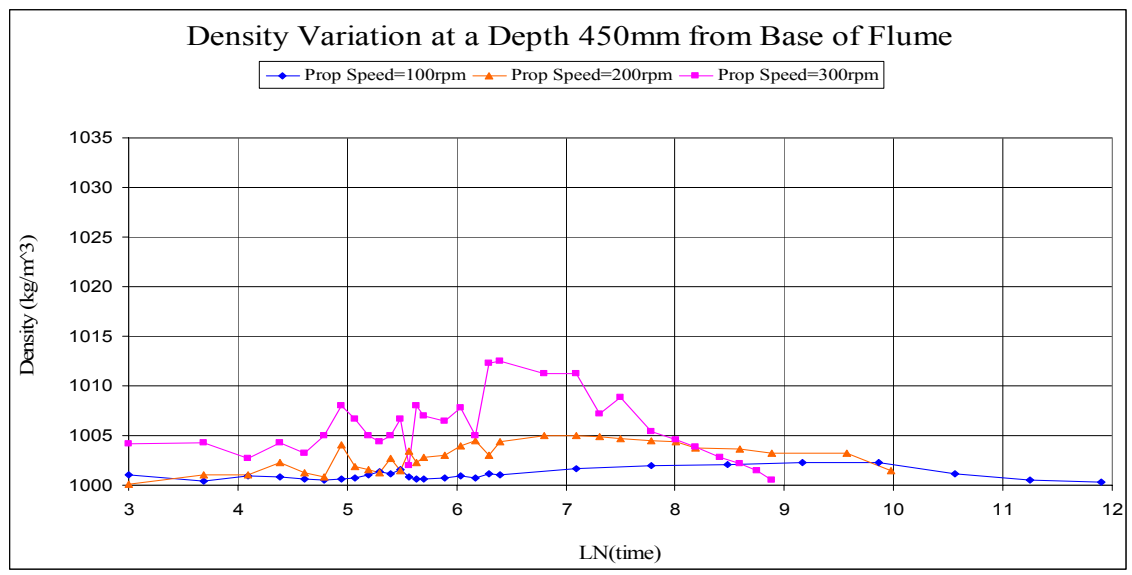

Figure 4: Density variation at a depth of $450 \mathrm{~mm}$ from base of flume.

Though no interfacial layer was observed in the current study yet it was necessary to establish an equation for predicting the temporal variation of density at the interfacial layer point to allow a comparison with the existing equation as follows:

where

$$
\mathrm{S} / \mathrm{S}_{\max }=\mathrm{bLN}(\mathrm{t})+\mathrm{a}
$$

$$
\begin{aligned}
& \mathrm{a}=0.3476+1.05 * 10^{-5} \mathrm{Re}-0.001349 \mathrm{Ri}_{\mathrm{b}}+1.638 \mathrm{Fr}_{\mathrm{D}} \\
& \mathrm{b}=-0.7007+2.38 * 10-6 \mathrm{Re}+0.00027 \mathrm{Ri}_{\mathrm{b}}-0.2398 \mathrm{Fr}_{\mathrm{D}}
\end{aligned}
$$

Following a similar pattern, all the $\mathrm{S}$ values (i.e. local density - freshwater density) were non-dimensionalized by dividing with the corresponding $\mathrm{S}_{\max }$ values (i.e. saltwater density - freshwater density) and the ratios were plotted on the vertical axis. The corresponding natural logarithm values of time intervals $(\mathrm{t}$ in seconds) were determined and plotted on the horizontal axis. Figure 5 shows the temporal development of density at the interfacial layer point for the propeller speed of $300 \mathrm{rpm}$. A regression analysis for the data was done to find out the best fit curve and is being represented below in mathematical form:

$$
\mathrm{S} / \mathrm{S}_{\max }=-0.3298 \mathrm{LN}(\mathrm{t})+2.8755\left(\mathrm{R}^{2}=0.9752\right)
$$

\subsection{Influence of propeller clearance on saltwater erosion}

Experimental tests were also performed to study the effect of propeller-tip to saltwater surface clearance. Propeller rig was designed in such a way so that the height of the propeller above the saltwater could be adjusted at any clearance. During this phase of research activity tests were carried out at two different clearances of propeller-tip from the saltwater layer, i.e. $85 \mathrm{~mm}$ and $185 \mathrm{~mm}$. For each test conductivity readings were taken at gradually increasing time intervals for four different depths, i.e. $50 \mathrm{~mm}, 200 \mathrm{~mm}, 300 \mathrm{~mm}$ and $450 \mathrm{~mm}$ from the 
base of the flume. The section at which these measurements were taken was located at a distance 2.5 times the propeller diameter. The propeller was run at a constant speed of $200 \mathrm{rpm}$. The density values for each test were plotted against natural logarithm values of time intervals for each depth. Figure 6 shows density variation for propeller speed $200 \mathrm{rpm}$ at depth $200 \mathrm{~mm}$ from the base of the flume. It can be seen in Fig. 6 that erosion of saltwater for the clearance $185 \mathrm{~mm}$ started later than that for the clearance $85 \mathrm{~mm}$. It can be further noticed that the saltwater scoured at a slower rate for the former clearance. This happened because the propeller away from the saltwater had weaker erosive action.

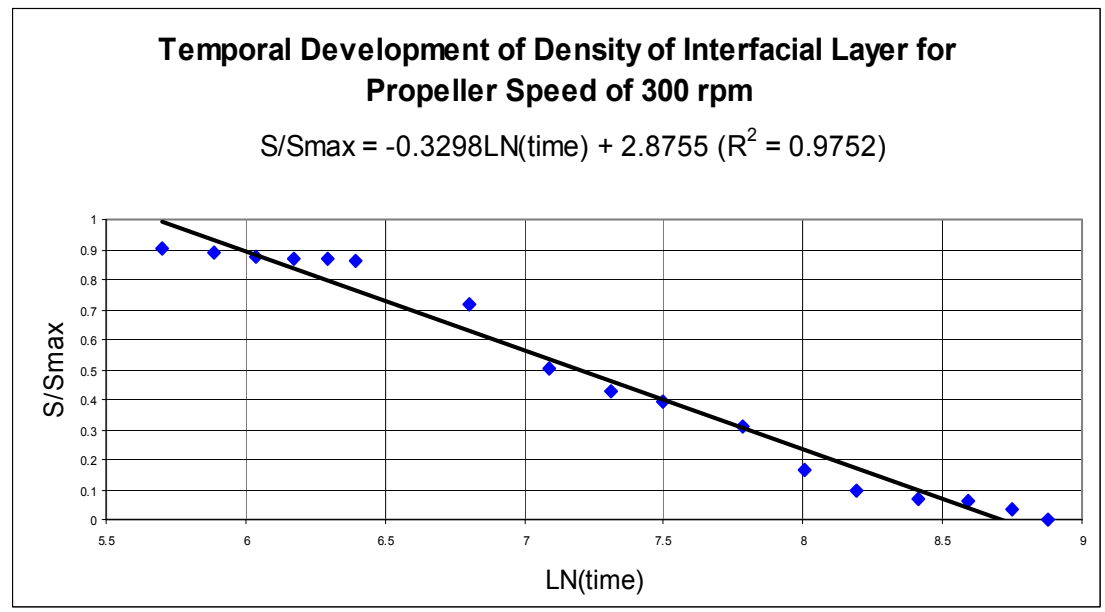

Figure 5: Temporal development of density of interfacial layer for propeller speed of $300 \mathrm{rpm}$.

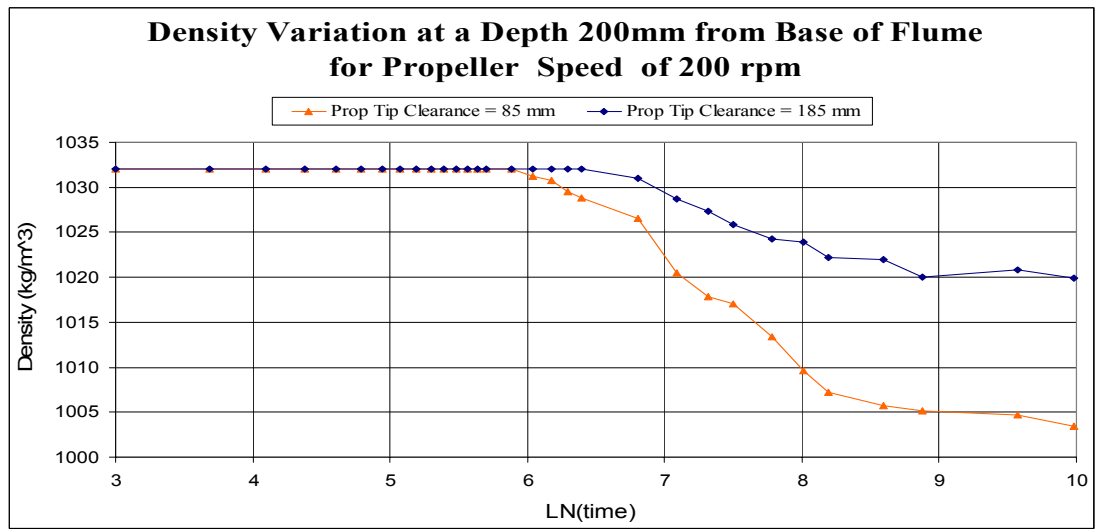

Figure 6: Density variation at a depth $200 \mathrm{~mm}$ from the base of flume for propeller speed of $200 \mathrm{rpm}$. 
Density variations with time at depths $300 \mathrm{~mm}$ and $450 \mathrm{~mm}$, in the flow were also compared and a similar trend was observed as shown in Fig. 7 and Fig. 8. Thus it can be concluded that an increase in propeller-tip clearance from the saltwater layer, reduces the rate of erosion of saltwater.

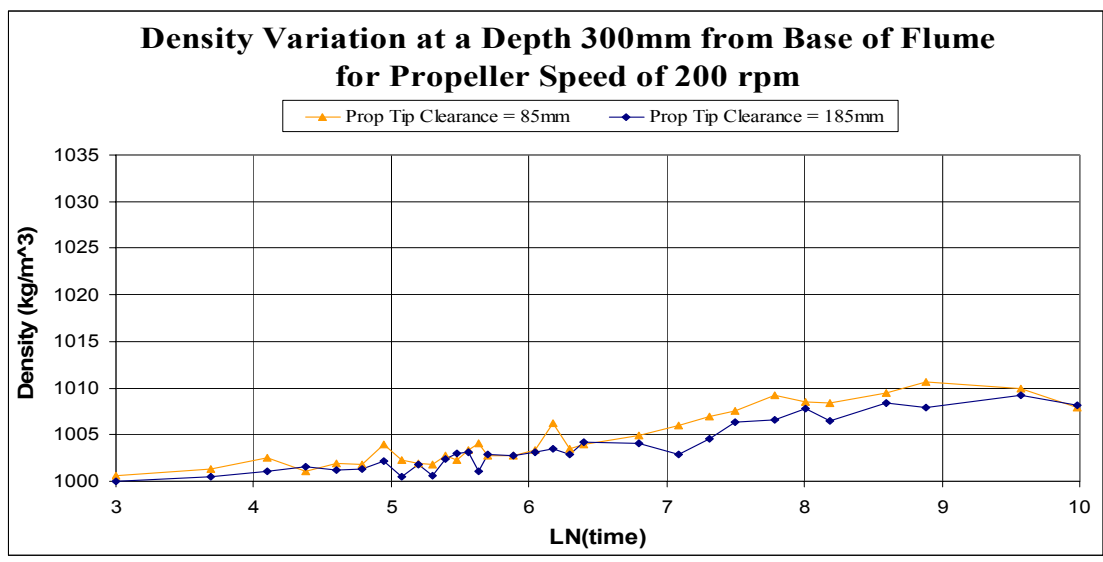

Figure 7: Density variation at a depth $300 \mathrm{~mm}$ from base of flume for propeller speed of $200 \mathrm{rpm}$.

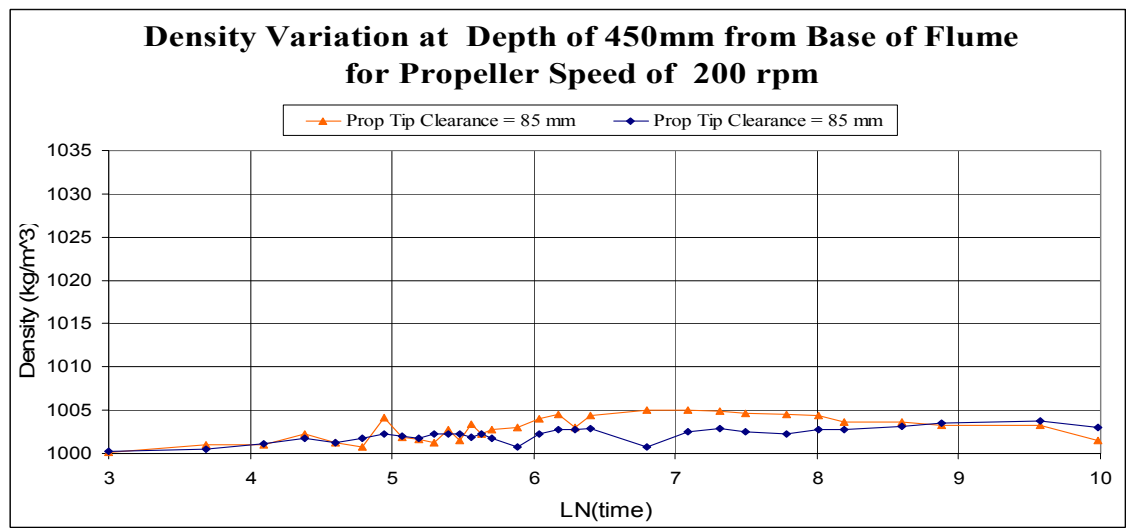

Figure 8: Density variation at depth of $450 \mathrm{~mm}$ from base of flume for propeller speed of $200 \mathrm{rpm}$.

\section{Conclusion}

From the experimental results it is concluded that propeller wash action is more effective to eliminate stratified flow system and it helps in proper mixing of freshwater and saltwater without any formation of interfacial layer. Experimental results show that high speed propeller wash is more effective. Under the 
influence of propeller wash on the stratified flow system, the density in the saltwater followed a reducing trend while that in the freshwater first increased to a maximum value and then started decreasing to minimum. It was also found that the erosive power of the wash decreased with an increase in propeller-tip clearance from the saltwater layer.

\section{References}

[1] Keulegan, G. H. (1966). 'The mechanism of an arrested saline wedge.' U.S. Army Engineer Waterways Experiment Station.' Vicksburg, Mississippi.

[2] Arita, M. \& Jirka G.H. (1987). 'Two - layer model of saline wedge. I: Entrainment \& interfacial friction.' J. of Hyd. Eng. Vol. 113 No. 10

[3] Grubert, J.P. (1989). 'Interfacial mixing in stratified channel flows.' J. of Hyd. Eng. Vol. 115. pp 887-905.

[4] Sorgard, E. (1991). 'A numerical 3 layered stationary salt wedge model.' J. of Geophysical Research.’ Vol. 96(C7). Pp 12739-12754.

[5] Curran, G. A. (1993). 'The effect of interfacial layer development on fresh water flows over an entrapped saline intrusion.' Ph.D. thesis, Department of Civil Engineering, The Queen's University of Belfast.

[6] Walker, S. A. (1996). 'The effect of time on interfacial mixing in density stratified flows.' Ph.D. thesis, Department of Civil Engineering, The Queen's University of Belfast. 